\title{
Knockdown of ARK5 Expression Suppresses Invasion and Metastasis of Gastric Cancer
}

\author{
Dehu Chen Guiyuan Liu Ning Xu Xiaolan You Haihua Zhou Xiaojun Zhao \\ Qinghong Liu
}

Department of General Surgery, Taizhou People's Hospital, The Fifth Affiliated Hospital of Nantong

University, Taizhou, China

\section{Key Words}

ARK5 • Gastric cancer • Epithelial-mensenchymal transition • mTOR

\begin{abstract}
Background/Aims: Gastric cancer (GC) is a common and lethal malignancy, and AMPactivated protein kinase-related kinase 5 (ARK5) has been discovered to promote cancer metastasis in certain types of cancer. In this study, we explored the role of ARK5 in GC invasion and metastasis. Methods: ARK5 and epithelial-mesenchymal transition (EMT)-related markers were determined by immunohistochemistry and western blot in GC specimens. Other methods including stably transfected against ARK5 into SGC7901 and AGS cells, western blot, migration and invasion assays in vitro and nude mice tumorigenicity in vivo were also employed. Results: The results demonstrated that ARK5 expression was increased and positively correlated with metastasis, EMT-related markers and poor prognosis in patients with GC. Knockdown of ARK5 expression remarkably suppressed GC cells invasion and metastasis via regulating EMT, rather than proliferation in vitro and in vivo. And knockdown of ARK5 expression in GC cells resulted in the down-regulation of the mTOR/p70S6k signals, Slug and SIP1. Conclusion: The elevated ARK5 expression was closely associated with cancer metastasis and patient survival, and it seemed to function in GC cells migration and invasion via EMT alteration, together with the alteration of the mTOR/p70S6k signals, Slug and SIP1, thus providing a potential therapeutic target for GC.

(C) 2017 The Author(s)

Published by S. Karger AG, Basel
\end{abstract}

\section{Introduction}

Gastric cancer (GC), ranking the fifth most common malignancy [1] and the third in all cancer-related deaths worldwide [2], remains a global cancer burden, with a considerable amount of morbidity and mortality [3]. Metastasis, a major cause of governing cancer patient mortality, is a complex and multistep process that is still unresolved, despite improvements in surgical treatment and chemotherapy during past decades [4]. Consequently, identification

D. Chen and G. Liu contributed equally to this paper 
of novel markers participation in the metastatic progression of GC, and understanding of the mechanisms responsible for GC invasion and metastasis, may provide potential therapeutic targets.

More recently, there are accumulating evidence that epithelial-mesenchymal transition (EMT) contributes to cancer cells invasion and metastasis [5-9]. The term EMT refers to a transdifferentiation process of epithelial cells into mesenchymal cells, in which epithelial cells shed certain features (such as cellular adherence and absence of motility), and gain mesenchymal characteristics (such as motility and invasiveness) [10]. During EMT, the hallmark is the loss of the adhesion molecule biomarker E-cadherin, together with the upregulated expression of Vimentin or N-cadherin. This switch in cell behaviour is controlled by crucial transcription factors, including Slug, Snail, ZEB, SIP1 and Twist, the functions of which are performed at transcriptional level; as well as non-transcriptional factors, such as signaling pathways responding to extracellular cues. As already reported, certain signaling pathways involved in EMT include the AKT/mTOR, Notch, Wnt, TGF- $\beta$ and AMP-activated protein kinase (AMPK) pathways $[5,11,12]$.

ARK5, known as the fifth member of the human AMPK family, is unique among AMPK catalytic subunit family members on account of its activation directly mediated by Aktdependent phosphorylation at serine residue on the regulatory domain [13]. Akt, one of the most commonly pathways in various types of cancers, is regarded as an important element in facilitating cancer cells proliferation, oncogenesis, survival, invasion and metastasis [14], and ARK5 is a crucial effector of these actions of Akt. Notably, recent investigations reveal that ARK5 is a critical molecule in promoting the invasion and metastasis of cancer cells in certain human malignancies [15, 16], including those of hepatocellular carcinoma [17], colorectal cancer [18], glioma [19] and non-small cell lung cancer [20]. More importantly, the high expression level of ARK5 in cancer tissues correlates with advanced clinical stage, enhanced metastatic potential and shortened patient survival $[17,19,20]$, indicating that ARK5 may be a valuable prognostic marker in a variety of human malignancies. More recently, an interesting finding demonstrates that ARK5 expression is closely associated with more aggressive cancer characteristics and a poor prognosis for patients with GC [21], despite its actual role and the underlying mechanism involved in GC invasion and metastasis have not yet been elucidated.

Based on the discussion mentioned above, we investigated in the current study whether ARK5 is involved in GC invasion and metastasis, using clinical samples of patients, human GC cell lines and nude mice.

\section{Materials and Methods}

\section{Patients selection and tissue preparation}

A total of 82 patients diagnosed with gastric adenocarcinoma who underwent radical gastrectomy at our hospital from January 2011 to March 2012 were enrolled in this study. Among them, fresh tissues of 25 cases were also assessed by western blot for ARK5 protein. None of them received either preoperative chemotherapy or radiotherapy. Primary lesion and corresponding non-cancerous tissue, identified by routine pathological observation, were embedded in paraffin blocks for various immunohistochemical stainings. All patients were followed up until November 2016, with a total of 9 cases (11.0\% patients) lost in follow-up period. Preoperative written consent was obtained from each patient in accordance with the Helsinki Declaration, and this study was also approved by the Ethics Committee of the Fifth Affiliated Hospital of Nantong University.

\section{Immunohistochemistry}

Expressions ofARK5,E-cadherin and Vimentinin specimenswere determinedby immunohistochemistry (IHC) as described previously [7]. We used antibodies against ARK5 (1:200, Abcam, UK), E-cadherin (1:200, CST, USA), and Vimentin (1:100, CST, USA). Sections were observed under the microscope. Two independent pathologists evaluated and scored the sections (double-blinded). The evaluation of staining result was graded as described previously [7], the percentage of staining cells scores $(0$ point $=0-5 \%$; 1 point $=6-25$ 


\section{Cellular Physiology Cell Physiol Biochem 2017;42:1025-1036 and Biochemistry Published onIIne: June 28, $2017 \quad \begin{aligned} & \text { DOI: 10.1159/000478685 } 2017 \text { The Author(s). Published by S. Karger AG, Basel } \\ & \text { www.karger.com/cpb }\end{aligned}$ \\ Chen et al.: ARK5 Regulates GC Cell Invasion and Metastasis}

$\% ; 2$ points $=26-50 \% ; 3$ points $=$ more than $50 \%)$ and the staining intensity scores $(0$ point $=$ negative; 1 point = weak intensity; 2 points = moderate intensity; 3 points = strong intensity) were summed. The sum scores $\geq 3$ points was considered as positive, while the sum scores $<3$ points was regarded as negative.

\section{Cell culture}

The human GC cell lines KATO-III, MKN28, SGC7901, AGS, MKN45 and MGC803 were obtained from the Type Culture Collection of the Chinese Academy of Sciences (Shanghai, China). KATO-III cells were maintained in $80 \%$ IMDM (ATCC, USA) supplemented with $20 \%$ fetal bovine serum (FBS) (Gibco). The other cell lines including MKN28, SGC7901, AGS, MKN45 and MGC803 were cultured in $90 \%$ RPMI-1640 (Gibco) supplemented with $10 \%$ FBS (Gibco). All cells were maintained in a humidified atmosphere of 37 ${ }^{\circ} \mathrm{C}$ containing $5 \% \mathrm{CO}_{2}$.

\section{Western blot}

Western blot was performed using standard technique as described previously [7]. Briefly, Cells were lysed in lysis buffer on ice. Equal amounts of protein were separated by SDS-PAGE and transferred to PVDF membrane (Millipore, USA). Membranes were blocked with $5 \%$ non-fat milk and then incubated with primary antibodies at $4{ }^{\circ} \mathrm{C}$ overnight. Secondary antibodies were incubated for $2 \mathrm{~h}$ at room temperature. Reactive bands were detected using the enhanced chemiluminescence detection kit (Thermo scientific, USA). The primary antibodies were: ARK5 rabbit pAb (1:500, Abcam, UK), p-mTOR rabbit mAb (1:1000, Abcam, UK), mTOR rabbit mAb (1:1000, Abcam, UK), p-p70S6K rabbit pAb (1:1000, Abcam, UK), p70S6K rabbit mAb (1:1000, Abcam, UK), Slug rabbit mAb (1:1000, CST, USA), SIP1 rabbit mAb (1:1000, CST, USA), Snail rabbit mAb (1:1000, CST, USA), ZEB1 rabbit mAb (1:1000, CST, USA), Twist mouse mAb (1:1000, Abcam, UK), E-cadherin rabbit mAb (1:1000, CST, USA), Vimentin rabbit mAb (1:1000, CST, USA), GAPDH mAb-HRP (1:5000, Bioworld Technology, USA).

\section{RNA interference and stably transfected clone selection}

Three shRNA oligonucleotides sequences for specifically targeting human ARK5 gene mRNA were designed using the RNAi designer. A nonspecific scrambled shRNA sequence was used as a control. The three shRNA sequence were as follows: the shRNA1 (siRNA1, 5'-GAATGATATCTTGTTGAGCTTGCTGCA-3'); the shRNA2 (siRNA2, 5'-CCAGATAAGCTTGCCACCATGGAAGGGGCCC-3'); the shRNA3 (siRNA3, 5'-CTAGAAGCTTCAGAAGGGGCCGCCGCG-3') [20]. The lentivirus with ARK5-gene was produced by cotransfection of 293T cells with Lipofiter (Hanbio, China). To establish stable ARK5-gene-konckdown cell lines, SGC7901 and AGS cells were infected with concentrated virus in serum-free medium. After $72 \mathrm{~h}$, cells were treated with $2 \mu \mathrm{g} / \mathrm{ml}$ puromycin. Puromycin-resistant clones were collected and expanded for further studies.

\section{Wound healing assay}

Cells were grown to $80 \%$ - $90 \%$ confluence in a 6-well plate, and a wound was created using a $10 \mu \mathrm{l}$ plastic tip to produce a straight line. Images were taken at $0 \mathrm{~h}$ and $48 \mathrm{~h}$ time points to monitor the wound healing process. The wound healing $=(0 \mathrm{~h}$ width $-48 \mathrm{~h}$ width $) / 0 \mathrm{~h}$ width $\times 100 \%$. Each experiment was performed in triplicate.

\section{Cell migration and invasion assays}

Cell migration and invasion assays were performed as described previously [7, 22]. In brief, cells migration and invasion were examined using $8-\mu \mathrm{m}$ pore size Transwell plates (Corning, USA). Cells $(3 \times$ $10^{5}$ ) in $200 \mu \mathrm{l}$ serum-free medium were added to the upper chamber containing an uncoated or Matrigel ${ }^{\mathrm{TM}}$ coated membrane (BD Biosciences, USA). The lower chamber was filled with $600 \mu$ medium containing 20 $\%$ fetal bovine serum as a chemoattractant. After $24 \mathrm{~h}$ of incubation, cells on the lower compartment were fixed in paraformaldehyde and stained with crystal violet. Migrated and invaded cells were counted under a microscope. Each experiment was performed in triplicate.

\section{Cell proliferation assay}

Cell counting kit-8 (CCK-8, Dojindo, Japan) was used to assess cell proliferation. Cells were seeded in each well of a 96 -well plate at a density $4 \times 10^{3}$ cells, and assessed at the indicated times $(0,24,48,72,96$ 
$\mathrm{h}$ after culture) according to the manufacture's instructions. The absorbance at $450 \mathrm{~nm}$ was measured to calculate cell growth rates.

\section{In vivo tumorigenesis}

Male nude athymic BALB/c mice (six-week-old) were used to examine tumorigenicity. To ascertain the role of ARK5 in tumor formation in vivo, the SGC7901 si Ctrl or ARK5 si group cells $\left(3 \times 10^{6}\right.$ cells in $0.2 \mathrm{ml}$ volume) were injected subcutaneously into nude mice followed by measurements of the tumor size at 5 -day intervals. Tumor size was calculated by the formula: volume $=(\text { short diameter })^{2} \times($ long diameter $) / 2$ [7]. At 30 days after injection, the nude mice were sacrificed, and the tumor mass were weighted. To explore the effects of ARK5 on tumor metastasis in vivo, The SGC7901 si Ctrl or ARK5 si group cells $\left(4 \times 10^{6}\right.$ cells in 0.2 $\mathrm{ml}$ volume) were injected intravenously into the tail vein of nude mice, respectively. After 30 days, the lungs of nude mice were observed by Siemens Inveon Micro PET/CT (Inveon MM platform, Siemens Preclinical Solutions, USA). Afterwards, the mice were sacrificed, and the number of metastatic foci in the lungs of nude mice was calculated. Subsequently, harvested lungs were subjected to further hematoxylin-eosin staining, western blot and IHC staining. The assays were performed using six nude mice per group, and all animal procedures were carried out in accordance with institutional guidelines.

\section{Statistical analysis}

The data are expressed as the means \pm SD. Statistical analyses of clinicopathologic features were done by Chi-square. Bivariate relations between categorical variables were performed by Spearman's rank correlation coefficient. Kaplan-Meier and Log-rank tests were used for survival analysis. For cell and animal experiments, data were analyzed by Student's t-test or One-Way ANOVA. All the analyses were performed using SPSS 21.0 software (SPSS Inc, USA). $P<0.05$ was considered statistically significant.

\section{Results}

Clinical significance of ARK5 in patients with GC

Initially, the expression of ARK5 protein was examined in GC tissues and corresponding noncancerous gastric tissues. The result revealed that the higher level of ARK5 protein was expressed in tumor samples than that in Paracancerous tissues (Fig. 1a). In addition, the expression levels of ARK5, E-cadherin and Vimentin were determined in primary lesion and in adjacent normal gastric mucosas of 82 patients with gastric adenocarcinoma by IHC

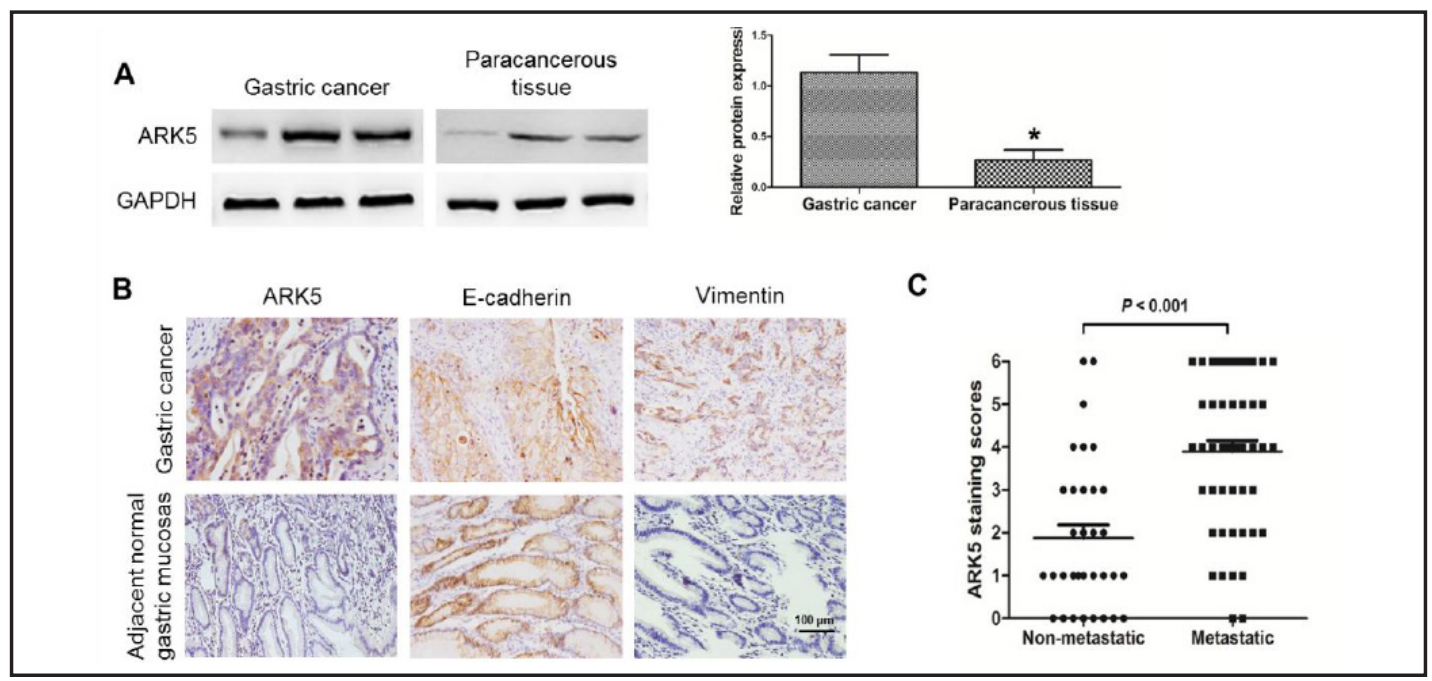

Fig. 1. Expressions of ARK5, E-cadherin and Vimentin in gastric cancer and adjacent normal gastric mucosas. (a) Western blot fotab.-r expression of ARK5 protein in gastric cancer. GAPDH protein was used as internal control. (b) Immunohistochemical stainings of ARK5, E-cadherin and Vimentin in gastric cancer tissues and adjacent normal gastric mucosas. (c) Scatterplots of the average staining scores of ARK5 expression in patients with or without metastasis. ${ }^{*} P<0.05$. Scale bar $=100 \mu \mathrm{m}$. 
Table 1. Expressions of ARK5, E-cadherin and Vimentin in gastric cancer and corresponding normal gastric mucosas

\begin{tabular}{|c|c|c|c|}
\hline Proteins & $\begin{array}{l}\text { Gastric cancer } \\
\text { tissues }\end{array}$ & $\begin{array}{l}\text { Gastric normal } \\
\text { mucosa tissues }\end{array}$ & P-value \\
\hline \multicolumn{4}{|l|}{ ARK5 } \\
\hline Positive & 48 & 29 & 0.003 \\
\hline Negative & 34 & 53 & \\
\hline \multicolumn{4}{|l|}{ E-cadherin } \\
\hline Positive & 33 & 56 & $<0.001$ \\
\hline Negative & 49 & 26 & \\
\hline \multicolumn{4}{|l|}{ Vimentin } \\
\hline Positive & 47 & 34 & 0.042 \\
\hline Negative & 35 & 48 & \\
\hline
\end{tabular}

Table 2. Correlation between ARK5, E-cadherin, Vimentin expression and clinicopathological features in gastric cancer

\begin{tabular}{|c|c|c|c|c|c|c|c|c|c|c|}
\hline \multirow{2}{*}{$\begin{array}{l}\text { Clinicopathological } \\
\text { features }\end{array}$} & \multirow{2}{*}{$\mathrm{n}$} & \multicolumn{3}{|c|}{ ARK5 } & \multicolumn{3}{|c|}{ E-cadherin } & \multicolumn{3}{|c|}{ Vimentin } \\
\hline & & + & - & $\mathrm{P}$-value & + & - & $\mathrm{P}$-value & + & - & P-value \\
\hline \multicolumn{11}{|l|}{ Age (yr) } \\
\hline$\geq 60$ & 57 & 33 & 24 & \multirow[t]{2}{*}{0.859} & 25 & 32 & \multirow[t]{2}{*}{0.313} & 34 & 23 & \multirow[t]{2}{*}{0.519} \\
\hline$<60$ & 25 & 15 & 10 & & 8 & 17 & & 13 & 12 & \\
\hline \multicolumn{11}{|l|}{ Gender } \\
\hline Male & 56 & 29 & 27 & \multirow[t]{2}{*}{0.069} & 24 & 32 & \multirow[t]{2}{*}{0.479} & 32 & 24 & \multirow[t]{2}{*}{0.963} \\
\hline Female & 26 & 19 & 7 & & 9 & 17 & & 15 & 11 & \\
\hline \multicolumn{11}{|l|}{ Tumor size $(\mathrm{cm})$} \\
\hline$\geq 5$ & 49 & 30 & 19 & \multirow[t]{2}{*}{0.547} & 18 & 31 & \multirow[t]{2}{*}{0.430} & 29 & 20 & \multirow[t]{2}{*}{0.677} \\
\hline$<5$ & 33 & 18 & 15 & & 15 & 18 & & 18 & 15 & \\
\hline \multicolumn{11}{|c|}{ Lauren's classification } \\
\hline Diffuse & 23 & 16 & 7 & \multirow[t]{2}{*}{0.206} & 9 & 14 & \multirow[t]{2}{*}{0.898} & 10 & 13 & \multirow[t]{2}{*}{0.114} \\
\hline Intestinal & 59 & 32 & 27 & & 24 & 35 & & 37 & 22 & \\
\hline \multicolumn{11}{|l|}{ Vascular invasion } \\
\hline With & 30 & 21 & 9 & \multirow[t]{2}{*}{0.110} & 9 & 21 & \multirow[t]{2}{*}{0.151} & 19 & 11 & \multirow[t]{2}{*}{0.403} \\
\hline Without & 52 & 27 & 25 & & 24 & 28 & & 28 & 24 & \\
\hline \multicolumn{11}{|c|}{ Lymphatic vessel invasion } \\
\hline With & 36 & 25 & 11 & \multirow[t]{2}{*}{0.076} & 11 & 25 & \multirow[t]{2}{*}{0.113} & 24 & 12 & \multirow[t]{2}{*}{0.130} \\
\hline Without & 46 & 23 & 23 & & 22 & 24 & & 23 & 23 & \\
\hline T stage & & & & & & & & & & \\
\hline $\mathrm{T}_{1}+\mathrm{T}_{2}$ & 37 & 15 & 22 & 0.003 & 20 & 17 & 0.021 & 15 & 22 & 0.005 \\
\hline $\mathrm{T}_{3}+\mathrm{T}_{4}$ & 45 & 33 & 12 & & 13 & 32 & & 32 & 13 & \\
\hline pTNM stage & & & & & & & & & & \\
\hline $\mathrm{I}+\mathrm{II}$ & 35 & 16 & 19 & 0.042 & 19 & 16 & 0.025 & 15 & 20 & 0.022 \\
\hline III + IV & 47 & 32 & 15 & & 14 & 33 & & 32 & 15 & \\
\hline Lymph node metast & & & & & & & & & & \\
\hline With $\left(\mathrm{N}_{1}+\mathrm{N}_{2}+\mathrm{N}_{3}\right)$ & 49 & 37 & 12 & $<0.001$ & 12 & 37 & $<0.001$ & 34 & 15 & 0.007 \\
\hline Without $\left(\mathrm{N}_{0}\right)$ & 33 & 11 & 22 & & 21 & 12 & & 13 & 20 & \\
\hline
\end{tabular}

analysis (Fig. 1b). As shown in Table 1, the expression levels of ARK5 and Vimentin in GC tissues were significantly higher than those in adjacent normal gastric mucosa $(p=0.003$ and $p=0.042$, respectively). In contrast, E-cadherin showed lower expression level in GC tissues $(p<0.001)$. A clinicopathological association analysis of the 82 patients with gastric adenocarcinoma revealed that the expression levels of ARK5, E-cadherin and Vimentin were closely correlated with T stage, pTNM stage and lymph node metastasis, respectively $(p<$ 0.05) (Table 2). Patients who developed lymph node metastasis showed significantly higher staining scores for ARK5 than those without metastasis ( $p<0.001)$ (Fig 1c), which indicates that ARK5 might play a role in metastasis. More intriguingly, patients with GC, respectively, with ARK5 positive, or Vimentin positive butE-cadherin negative were correlated significantly with shorter overall survival ( $p<0.05$, respectively) (Fig. 2). Additionally, Table 3 revealed the expression level of ARK5 was positively correlated with that of Vimentin ( $\mathrm{r}=0.225, p=$ 0.043 ), but inversely related with E-cadherin expression ( $r=-0.470, p<0.001)$ in GC tissues.

Down-regulation of ARK5 in GC cells

To identify the appropriate GC cell lines with high expression level of ARK5, we examined the expression level of ARK5 in the GC cell lines KATO-III, MKN28, SGC7901, AGS, MKN45 


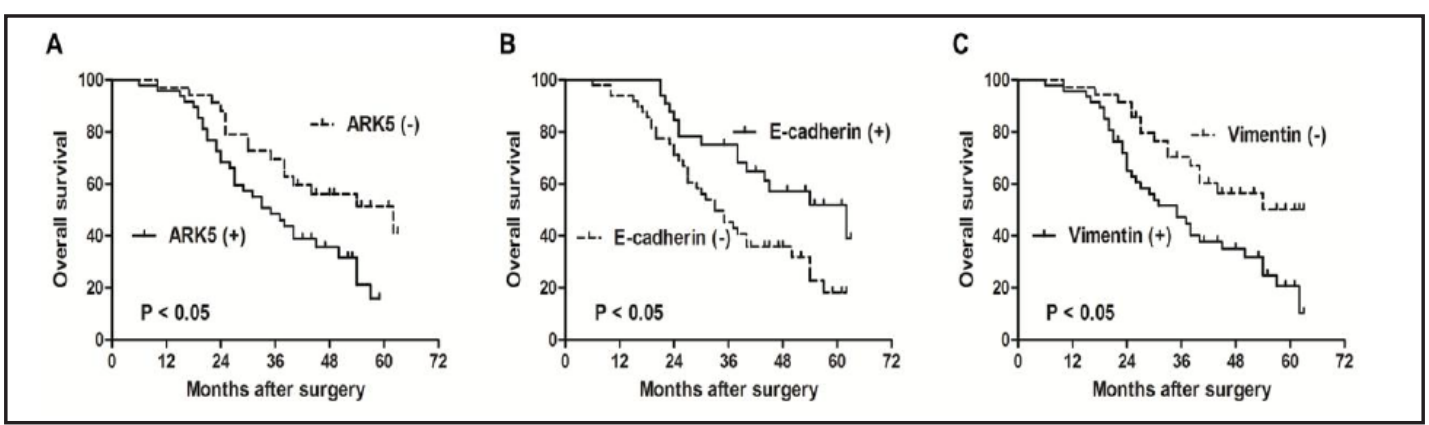

Fig. 2. Survival curves of gastric cancer patients with ARK5, E-cadherin or Vimentin expression. (a) The overall survival of ARK5 marker in patients with gastric cancer. (b) The overall survival of E-cadherin marker in patients with gastric cancer. (c) The overall survival of Vimentin marker in patients with gastric cancer.

Table 3. Spearman relation analysis among expressions of ARK5, E-cadherin and Vimentin in GC tissues

\begin{tabular}{cllll}
\hline & $\begin{array}{l}\text { ARK5 } \\
\text { Positive }\end{array}$ & Negative & r & P-value \\
\hline E-cadherin & & & & \\
Positive & 10 & 23 & -0.470 & $<0.001$ \\
Negative & 38 & 11 & & \\
Vimentin & & 15 & 0.225 & 0.043 \\
Positive & 32 & 19 & & \\
Negative & 16 & & & \\
\hline
\end{tabular}

and MGC803. SGC7901 and AGS cells showed the highest levels of ARK5 protein expression (Fig. 3a), and were selected for further studies.

To further confirm the role of ARK5 in GC cells metastasis, three RNA interference expression vectors (siRNA1, siRNA2 and siRNA3) were designed to target human ARK5 in GC cell lines SGC7901 and AGS; meanwhile, a scrambled sequence (si Ctrl) was employed as a control. Obviously, the most widely reduced expression of the siRNA3 group was observed by western blot (Fig. 3b), which demonstrated that the siRNA3 group had the most efficient knockdown in SGC7901 and AGS cells. Therefore, the GC cell lines SGC7901 and AGS siRNA3 group cells were employed for follow-up assays.

ARK5 modulates EMT, together with the alteration of the mTOR/p70S6k signals, Slug and SIP1 in GC cells

It is acknowledged that E-cadherin, Vimentin and transcription factors are specific biomarkers for the EMT process [5]. To ascertain whether ARK5 knockdown facilitates EMT in GC cell lines, the expression levels of these markers were detected by western blot in SGC7901 and AGS cells (Blank, si Ctrl and ARK5 si) (Fig. 4). Remarkably, compared with the Blank and the si Ctrl groups, the expression levels of ARK5, Slug, SIP1 and Vimentin in the ARK5 si group dramatically decreased. Conversely, the expression level of E-cadherin significantly increased. The expression levels of Snail, ZEB1 and Twist didn't significantly varied (Fig. 4). Since the mTOR signaling pathway is one of the major pathways regulating the migration and invasion of cancer cells [23]. We focused on mTOR/p70S6K (p70S6k, a downstream effector of mTOR) signals. Clearly, a decrease in the levels of p-mTOR and p-p70S6k, but not the total amounts of these proteins, was observed in the ARK5 si group (Fig. 4). Based on the above results of this section, it was demonstrated that knockdown of ARK5 expression modulated EMT in GC cells, resulting in the down-regulation of the mTOR/ p70S6k signals, Slug and SIP1.

\section{ARK5 promotes GC cells migration and invasion in vitro}

To prove the influence of ARK5 reduction on the migration and invasion capacities of GC cells, the transwell chambers and the wound healing assays were used. As shown in Fig. 5a-b, the migratory and invasive activities were significantly impaired in SGC7901 and AGS 


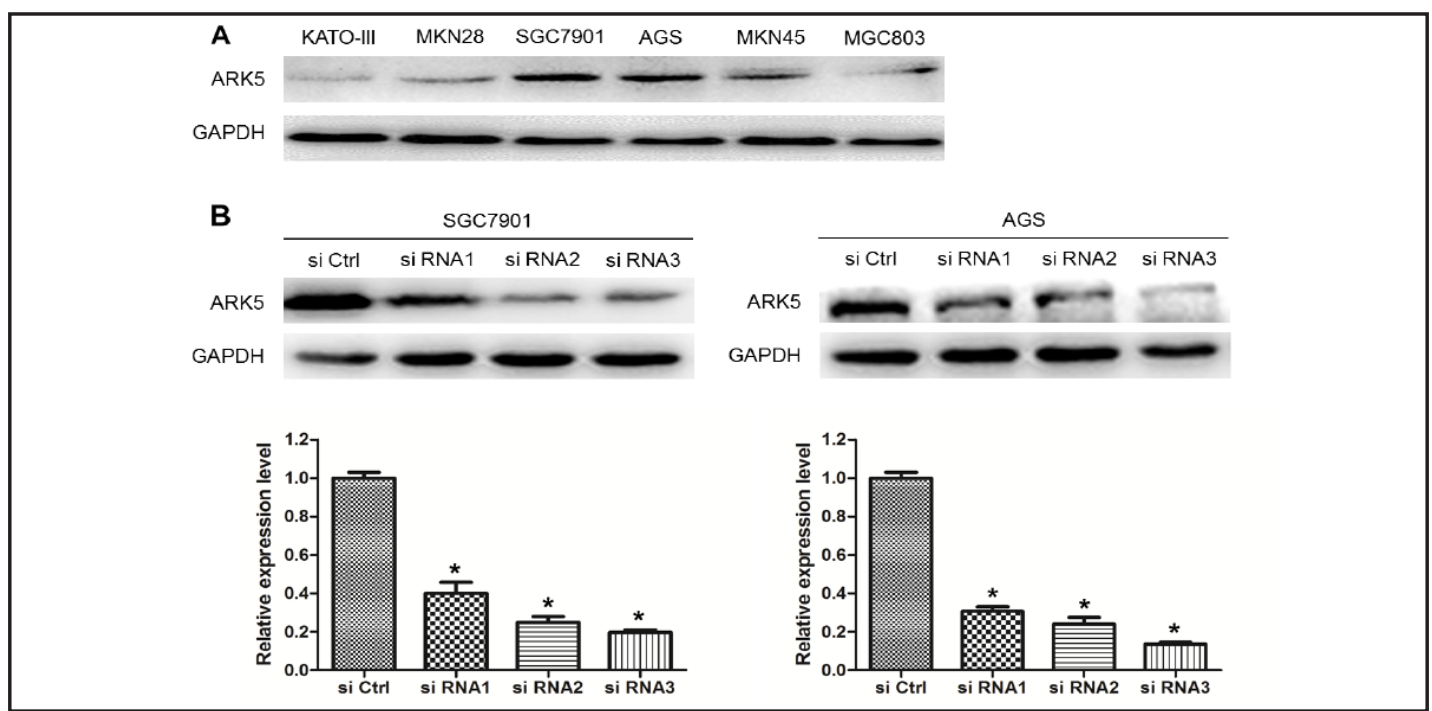

Fig. 3. Expression of ARK5 in gastric cancer cells and knockdown of ARK5 in SGC7901 and AGS cells. (a) Relative expression of ARK5 protein in gastric cancer cell lines (KATO-III, MKN28, SGC7901, AGS, MKN45 and MGC803) was detected by western blot. (b) The interfering efficiency of ARK5 transfected with lentiviral expression vectors (si Ctrl, siRNA1, siRNA2 and siRNA3) in SGC7901 and AGS cells was determined by western blot. GAPDH protein was used as internal control. Images are representative of three independent experiments $(\mathrm{n}=3) .{ }^{*} P<0.05$.

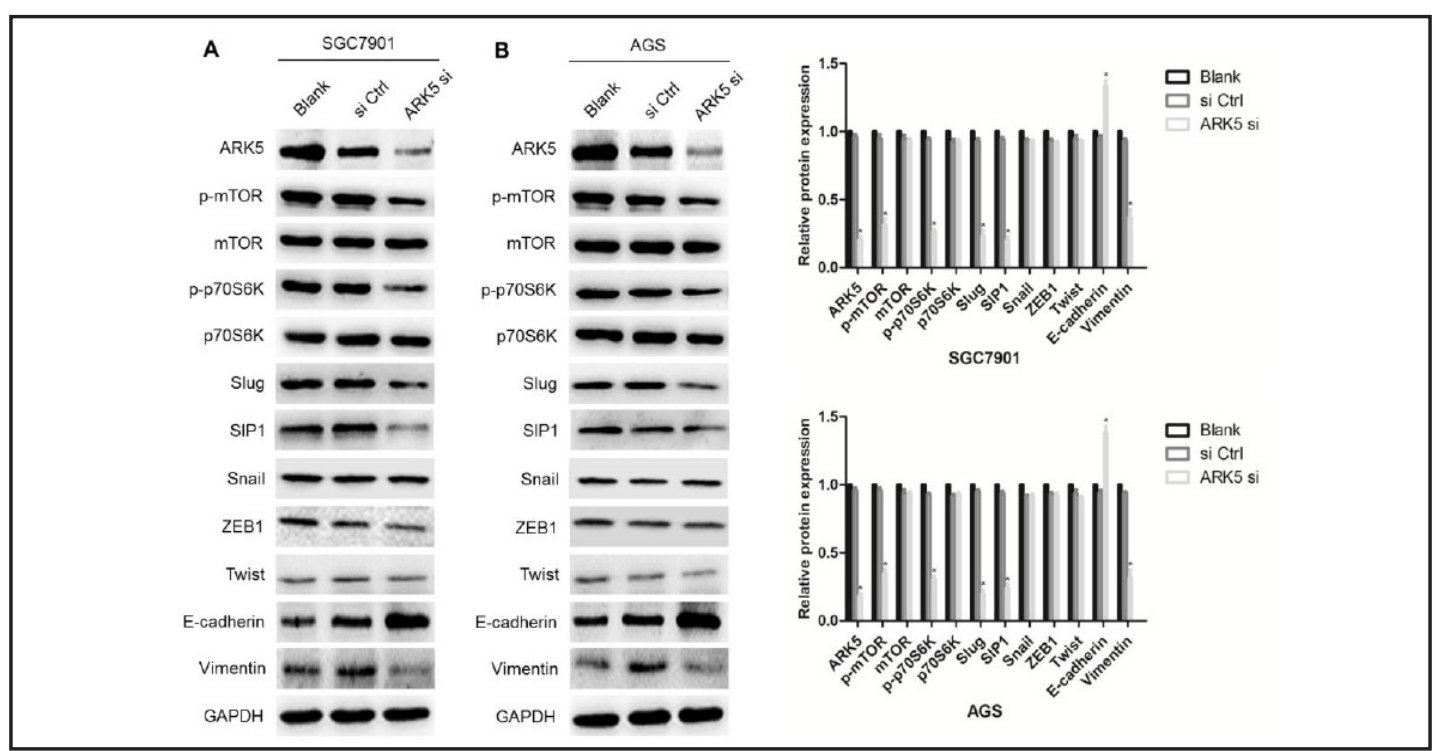

Fig. 4. Suppression effect of ARK5 expression on EMT, mTOR/p70S6k signals and transcription factors in SGC7901 and AGS cells. (a,b) Expressions of ARK5, p-mTOR, p-p70S6k, transcription factors (Slug, SIP1, Snail, ZEB1, Twist), E-cadherin and Vimentin were examined in SGC7901 and AGS cells silencing of ARK5 expression by western blot. GAPDH protein was used as internal control. Images are representative of three independent experiments $(\mathrm{n}=3)$. $* P<0.05$.

ARK5 si groups cells $(P<0.05)$. Besides, similar results revealed in the wound healing assay that ARK5 si groups cells displayed observably slower migration towards the wound space compared with the Blank and si Ctrl groups cells (Fig. 5c). Taken together, these results suggested that ARK5 played an important part in the migration and invasion of GC cells in vitro. To determine whether ARK5 could affect cell proliferation in vitro, the cellular growth 
Fig. 5. Effects of knockdown of ARK5 expression on gastric cancer cells migration and invasion. (a,c) Silencing effects of ARK5 expression on the migration ability of SGC7901 and AGS cells by transwell assay and wound healing assay. (b) Suppression effects of ARK5 expression on the invasion ability of SGC7901 and AGS cells by transwell assay. Images are representative of three independent experiments $(\mathrm{n}=3)$. ${ }^{*} P<0.05$. Scale bar $=100 \mu \mathrm{m}$.

Fig. 6. Effects of knockdown of ARK5 expression on gastric cancer cell proliferation in vitro and in vivo. (a) Effects of knockdown of ARK5 expression on the proliferation ability of SGC7901 and AGS cells by CCK8 assay. (b) Influences of knockdown of ARK5 expression on the proliferation of SGC7901 cells in nude mice. Tumor volume was calculated every 5 days. Tumors were weighed at 30 days after injection. $\mathrm{n}=6$ in per group. Images are representative of three independent experiments $(n=3)$.

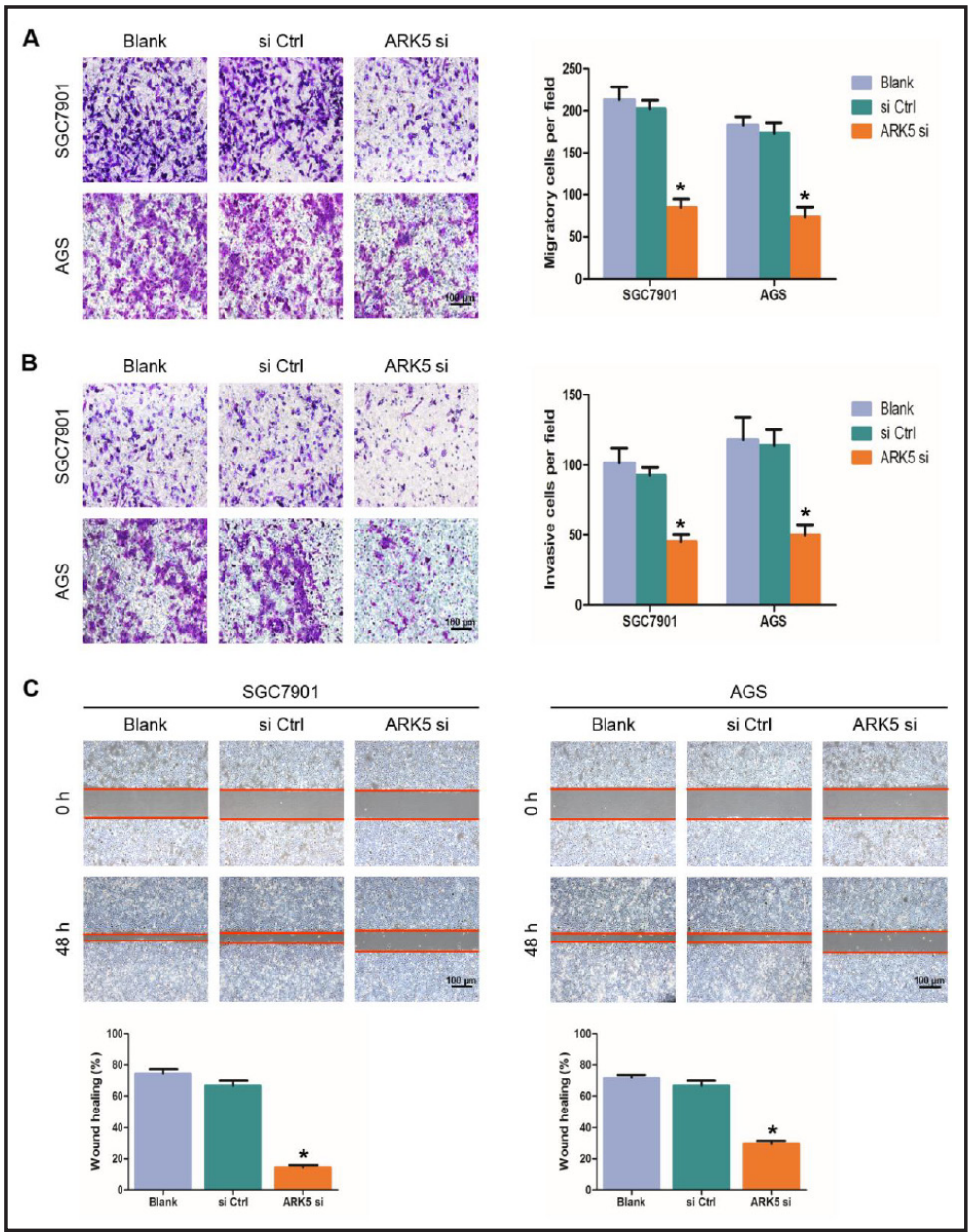

A

B
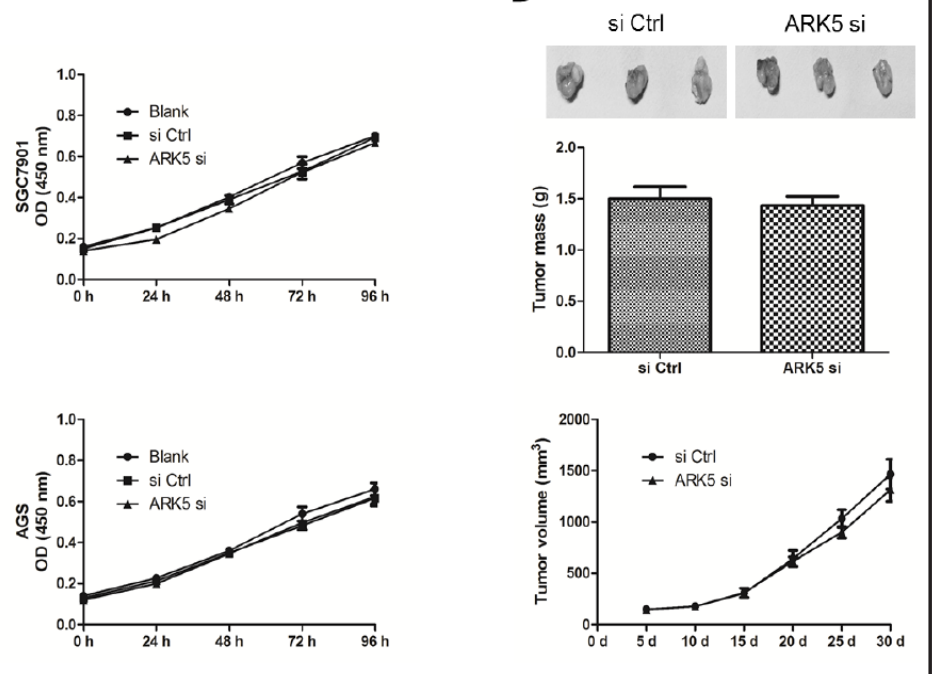

rates in three groups were compared. As shown in Fig. 6a, three groups exhibited similar proliferation rates, suggesting that knockdown of ARK5 expression had no significant effects on GC cells proliferation.

\section{KARGER}




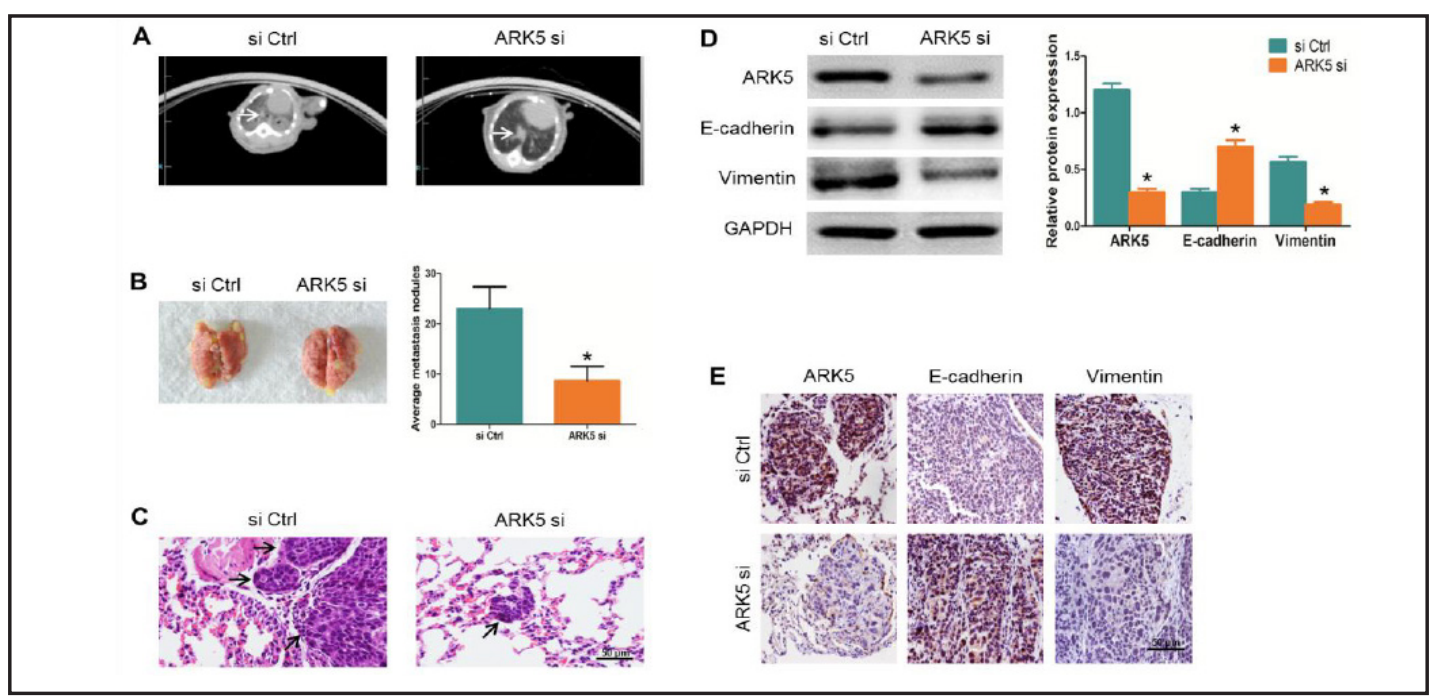

Fig. 7. Influence of ARK5 knockdown on the metastasis of SGC7901 cells in vivo. (a) Observation on the lungs of nude mice via Micro-PET/CT scanning. (b) Average metastasis nodules were calculated in lungs of nude mice. (c) Metastatic tumor nodules (shown by black arrows) in the lungs of nude mice were histologically determined via HE staining. (d,e) Expressions of ARK5, E-cadherin and Vimentin in metastasis nodules in the lungs of nude mice were identified by western blot and immunohistochemistry. GAPDH protein was used as internal control. $\mathrm{n}=6$ in per group. Images are representative of three independent experiments (n =3). ${ }^{*} P<0.05$. Scale bar $=50 \mu \mathrm{m}$.

\section{ARK5 is essential for SGC7901 cells metastasis in vivo}

To ascertain whether ARK5 could affect tumor formation in vivo, SGC7901 cells (si Ctrl or ARK5 si) were injected subcutaneously into nude mice. As shown in Fig. 6b, the weight and the volume of tumor were almost identical between the two groups, suggesting that ARK5 was not essential for tumor formation in SGC7901 cells. To determine whether knockdown of ARK5 expression could suppress GC cells metastasis in vivo. SGC7901 cells (si Ctrl or ARK5 si) were injected into the tail vein of nude mice, respectively. The lungs of mice were scanned through Micro PET/CT exposure, and the number of lung metastatic foci was calculated 30 days later. Obviously, the model of lung metastatic foci in nude mice was found to be successfully established through the Micro PET/CT scanning (Fig. 7a). Afterwards, a significantly less number of lung metastasis foci could be observed in the ARK5 si group compared with the si Ctrl group (Fig. 7b-c). Furthermore, consistent with the finding in vitro, the expression levels of ARK5 and Vimentin decreased, combined with E-cadherin upregulation, in metastasis nodules in the lungs of the ARK5 si group, by western blot and IHC assays (Fig. 7d-e). Consequently, these observations suggested that knockdown of ARK5 expression could inhibit GC SGC7901 cells metastasis in vivo.

\section{Discussion}

It is interesting to note, that numerous genetic changes are relation to the invasion and metastasis of cancer cells $[23,24]$. Hence, in search for key regulators in the metastatic process of GC is very meaningful. AMPK, at present, is already known to act as a tumor suppressor through interacting with a network of complex structures in cancer cells. ARK5, one of members of AMPK family, is considered as be involved in cancer invasion and metastasis as a result of its great sequence homology to the catalytic domain of AMPK [25]. ARK5 was first deemed as a tumor malignancy-associated factor in Akt-induced cell migration activity in human pancreatic and colon cancer cells [13]. As already reported, ARK5, as a downstream substrate of Akt, was directly activated by phosphorylation at its 
$\mathrm{Ser}^{600}$, and therefore resulting in stimulation of cancer cells invasion and metastasis [15]. To date, ARK5 has been confirmed to facilitate cancer metastasis through the induction of EMT or the activation of matrix metalloproteinase 2 (MMP2) and MMP9 in certain human malignancies [20, 26-28]. However, few investigations about the effect of ARK5 on the GC invasion and metastasis have been reported.

In this study, our data showed that ARK5 was frequently overexpressed in GC tissues, as well as related with clinical metastasis and patient survival, implying that high expression of ARK5 may be a useful diagnostic marker for GC. To better evaluate the potential role of the ARK5 gene in GC invasion and metastasis, we performed recombinant plasmid vectors and transfected it into GC cell lines SGC7901 and AGS. The results revealed that knockdown of ARK5 expression significantly decreased the invasive and metastatic potential of GC cells in vitro and in vivo. Furthermore, in the section of nude mice metastatic model, it is noteworthy that the model construction does not conform to the criterion of physiologic metastatic model. However, as previously reported, this metastatic model has been employed to determine the invasive and metastatic potential of cancer cells [7, 29, 30], in that this model is capable of imitating the overflow of cancer cells from blood vessels into the target organ, which is considered as a critical step in the metastatic process [31].

EMT is widely accepted to be closely associated with cancer cells invasion and metastasis [32]. To ascertain whether ARK5 promotes GC cells invasion and metastasis through the induction of EMT, we explored the role of ARK5 in the expression levels of E-cadherin and Vimentin in GC cells. In the light of ARK5 knockdown, E-cadherin expression was upregulated, together with Vimentin expression down-regulated. Besides, ARK5 expression was positively correlated with Vimentin expression in GC tissues, but negatively associated with E-cadherin expression. As a result of these findings, it seems reasonable that the effects of ARK5 on GC cells invasion and metastasis may be attributed to its induction of EMT.

Akt/mTOR/p70S6k signaling pathway is of great importance in the induction of EMT [33, 34]. Our present results showed that down-regulation of mTOR and p70S6k (a downstream mediator of mTOR) phosphorylation was caused by ARK5 (a direct downstream effector of Akt) knockdown. Combined with a previous finding that mTOR was directly phosphorylated by Akt [35], it seems likely that certain factors modulating mTOR phosphorylation is phosphorylated by ARK5 for mTOR phosphorylation. Perhaps, ARK5 may directly induce mTOR phosphorylation at a site. In view of its essential complexity and greater importance of mTOR pathway, these issues should be further examined in the future. In addition, our data showed the reduction of transcription factors (Slug and SIP1), might contribute EMT owing to the indirect induction of ARK5 knockdown. Collectively, knockdown of ARK5 expression suppresses invasion and metastasis of GC, together with the down-regulation of the MTOR/ p70S6k signals, Slug and SIP1.

In summary, our study demonstrated that the elevated expression level of ARK5 closely correlated with poor pathological characteristics in patients with GC, and that knockdown of ARK5 expression suppressed GC cells invasion and metastasis, coupling with the downregulation of the mTOR/p70S6k signals, Slug and SIP1. Further exploration is nonetheless required to deepen the understanding of the mechanism(s) through which ARK5 facilitates GC cells invasion and metastasis, with the purpose of developing a novel therapeutic target.

\section{Acknowledgements}

This work was supported in part by funding from Science Foundation of Taizhou People's Hospital (Grant no.ZL201718) and the National Natural Science Foundation of China (Grant no.81600434).

\section{Disclosure Statement}

The authors declare they have no conflicts of interest. 


\section{Cellular Physiology Cell Physiol Biochem 2017;42:1025-1036

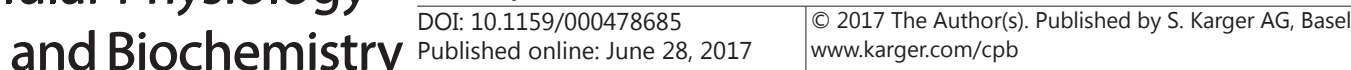

Chen et al.: ARK5 Regulates GC Cell Invasion and Metastasis

\section{References}

1 Ferlay J, Soerjomataram I, Dikshit R, Eser S, Mathers C, Rebelo M, Parkin DM, Forman D, Bray F: Cancer incidence and mortality worldwide: Sources, methods and major patterns in GLOBOCAN 2012. Int J Cancer 2015;136:E359-386.

2 Fock KM: Review article: the epidemiology and prevention of gastric cancer. Aliment Pharmacol Ther 2014;40:250-260.

-3 Torre LA, Bray F, Siegel RL, Ferlay J, Lortet-Tieulent J, Jemal A: Global Cancer Statistics, 2012. CA Cancer J Clin 2015;65:87-108.

4 Catalano V, Labianca R, Beretta GD, Gatta G, de Braud F, Van Cutsem E: Gastric cancer. Crit Rev Oncol Hematol 2009;71:127-164.

-5 Lamouille S, Xu J, Derynck R: Molecular mechanisms of epithelial-mesenchymal transition. Nat Rev Mol Cell Biol 2014;15:178-196.

-6 Xian X, Huang L, Zhang B, Wu C, Cui J, Wang Z: WIN 55,212-2 Inhibits the Epithelial Mesenchymal Transition of Gastric Cancer Cells via COX-2 Signals. Cell Physiol Biochem 2016;39:2149-2157.

7 Chen DH, Yu JW, Wu JG, Wang SL, Jiang BJ: Significances of contactin-1 expression in human gastric cancer and knockdown of contactin-1 expression inhibits invasion and metastasis of MKN45 gastric cancer cells. J Cancer Res Clin Oncol 2015;141:2109-2120.

8 Wu K, Shen B, Jiang F, Xia L, Fan T, Qin M, Yang L, Guo J, Li Y, Zhu M, Du J, Liu Y: TRPP2 Enhances Metastasis by Regulating Epithelial-Mesenchymal Transition in Laryngeal Squamous Cell Carcinoma. Cell Physiol Biochem 2016;39:2203-2215.

-9 Zhou Q Zheng X, Chen L, Xu B, Yang X, Jiang J, Wu C: Smad2/3/4 Pathway Contributes to TGF- $\beta$-Induced MiRNA-181b Expression to Promote Gastric Cancer Metastasis by Targeting Timp3. Cell Physiol Biochem 2016;39:453-466.

10 Heerboth S, Housman G, Leary M, Longacre M, Byler S, Lapinska K, Willbanks A, Sarkar S: EMT and tumor metastasis. Clin Transl Med 2015;4:6.

-11 Chen DH, Yu JW, Jiang BJ: Contactin 1: A potential therapeutic target and biomarker in gastric cancer. World J Gastroenterol 2015;21:9707-9716.

12 Wu W, Ding H, Cao J, Zhang W: FBXL5 inhibits metastasis of gastric cancer through suppressing Snail1. Cell Physiol Biochem 2015;35:1764-1772.

13 Suzuki A, Kusakai G, Kishimoto A, Lu J, Ogura T, Lavin MF, Esumi H: Identification of a novel protein kinase mediating Akt survival signaling to the ATM protein. J Biol Chem 2003;278:48-53.

14 Warfel NA, Kraft AS: PIM kinase (and Akt) biology and signaling in tumors. Pharmacol Ther 2015;151:4149.

15 Suzuki A, Kusakai G, Kishimoto A, Shimojo Y, Miyamoto S, Ogura T, Ochiai A, Esumi H: Regulation of caspase- 6 and FLIP by the AMPK family member ARK5. Oncogene 2004;23:7067-7075.

16 Kusakai G, Suzuki A, Ogura T, Kaminishi M, Esumi H: Strong association of ARK5 with tumor invasion and metastasis. J Exp Clin Cancer Res 2004;23:263-268.

17 Cui J, Yu Y, Lu GF, Liu C, Liu X, Xu YX, Zheng PY: Overexpression of ARK5 is associated with poor prognosis in hepatocellular carcinoma. Tumour Biol 2013;34:1913-1918.

18 Kusakai G, Suzuki A, Ogura T, Miyamoto S, Ochiai A, Kaminishi M, Esumi H: ARK5 expression in colorectal cancer and its implications for tumor progression. Am J Pathol 2004;164:987-995.

-19 Lu S, Niu N, Guo H, Tang J, Guo W, Liu Z, Shi L, Sun T, Zhou F, Li H, Zhang J, Zhang B: ARK5 promotes glioma cell invasion, and its elevated expression is correlated with poor clinical outcome. Eur J Cancer 2013;49:752-763.

20 Chen P, Li K, Liang Y, Li L, Zhu X: High NUAK1 expression correlates with poor prognosis and involved in NSCLC cells migration and invasion. Exp Lung Res 2013;39:9-17.

21 Ye XT, Guo AJ, Yin PF, Cao XD, Chang JC: Overexpression of NUAK1 is associated with disease-free survival and overall survival in patients with gastric cancer. Med Oncol 2014;31:61.

22 Zhou X, Ye F, Yin C, Zhuang Y, Yue G, Zhang G: The Interaction Between MiR-141 and lncRNA-H19 in Regulating Cell Proliferation and Migration in Gastric Cancer. Cell Physiol Biochem 2015;36:1440-1452.

23 Su JL, Yang CY, Shih JY, Wei LH, Hsieh CY, Jeng YM, Wang MY, Yang PC, Kuo ML: Knockdown of contactin-1 expression suppresses invasion and metastasis of lung adenocarcinoma. Cancer Res 2006;66:2553-2561. 


\section{Cellular Physiology Cell Physiol Biochem 2017;42:1025-1036

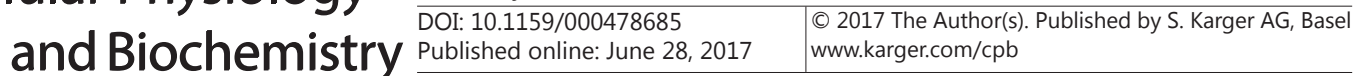 \\ Chen et al.: ARK5 Regulates GC Cell Invasion and Metastasis}

24 Xiang XJ, Deng J, Liu YW, Wan LY, Feng M, Chen J, Xiong JP: MiR-1271 Inhibits Cell Proliferation, Invasion and EMT in Gastric Cancer by Targeting FOXQ1. Cell Physiol Biochem 2015;36:1382-1394.

25 Sun X, Gao L, Chien HY, Li WC, Zhao J: The regulation and function of the NUAK family. J Mol Endocrinol 2013;51:R15-22.

26 Suzuki A, Lu J, Kusakai G, Kishimoto A, Ogura T, Esumi H: ARK5 is a tumor invasion-associated factor downstream of Akt signaling. Mol Cell Biol 2004;24:3526-3535.

27 Zhang HY, Li JH, Li G, Wang SR: Activation of ARK5/miR-1181/HOXA10 axis promotes epithelialmesenchymal transition in ovarian cancer. Oncol Rep 2015;34:1193-1202.

-28 Obayashi M, Yoshida M, Tsunematsu T, Ogawa I, Sasahira T, Kuniyasu H, Imoto I, Abiko Y, Xu D, Fukunaga S, Tahara H, Kudo Y, Nagao T, Takata T: microRNA-203 suppresses invasion and epithelial-mesenchymal transition induction via targeting NUAK1 in head and neck cancer. Oncotarget 2016;7:8223-8239.

29 Huang J, Xiao D, Li G, Ma J, Chen P, Yuan W, Hou F, Ge J, Zhong M, Tang Y, Xia X, Chen Z: EphA2 promotes epithelial-mesenchymal transition through the Wnt/beta-catenin pathway in gastric cancer cells. Oncogene 2014;33:2737-2747.

-30 Johnson JL, Pillai S, Pernazza D, Sebti SM, Lawrence NJ, Chellappan SP: Regulation of Matrix Metalloproteinase Genes by E2F Transcription Factors: Rb-Raf-1 Interaction as a Novel Target for Metastatic Disease. Cancer Res 2012;72:516-526.

-31 Elkin M, Vlodavsky I: Tail vein assay of cancer metastasis. Curr Protoc Cell Biol 2001; Chapter 19:Unit 19.2.

32 Nieto MA, Huang RY, Jackson RA, Thiery JP: EMT: 2016. Cell 2016;166:21-45.

33 Ahmad A, Biersack B, Li Y, Kong D, Bao B, Schobert R, Padhye SB, Sarkar FH: Targeted Regulation of PI3K/ Akt/mTOR/NF-kappa B Signaling by Indole Compounds and their Derivatives: Mechanistic Details and Biological Implications for Cancer Therapy. Anticancer Agents Med Chem 2013;13:1002-1013.

-34 Cheng K, Hao M: Metformin Inhibits TGF-beta1-Induced Epithelial-to-Mesenchymal Transition via PKM2 Relative-mTOR/p70s6k Signaling Pathway in Cervical Carcinoma Cells. Int J Mol Sci 2016;17:E2000.

-35 Brunet A, Bonni A, Zigmond MJ, Lin MZ, Juo P, Hu LS, Anderson MJ, Arden KC, Blenis J, Greenberg ME: Akt promotes cell survival by phosphorylating and inhibiting a Forkhead transcription factor. Cell 1999;96:857-868. 